\title{
Le Portique
}

Revue de philosophie et de sciences humaines

$8 \mid 2001$

Nietzsche et le divin

\section{En ouverture}

Yves Ledure

\section{OpenEdition}

Journals

Édition électronique

URL : http://journals.openedition.org/leportique/206

DOI : 10.4000/leportique.206

ISSN : $1777-5280$

\section{Éditeur}

Association "Les Amis du Portique"

Édition imprimée

Date de publication : 1 septembre 2001

ISSN : 1283-8594

\section{Référence électronique}

Yves Ledure, «En ouverture », Le Portique [En ligne], 8| 2001, mis en ligne le 09 mars 2005, consulté le 12 avril 2021. URL : http://journals.openedition.org/leportique/206 ; DOl : https://doi.org/10.4000/ leportique.206

Ce document a été généré automatiquement le 12 avril 2021

Tous droits réservés 


\section{En ouverture}

\section{Yves Ledure}

1 Le centenaire de la mort de Nietzsche en 2000 a apporté, s'il en était encore besoin, la preuve de l'actualité de sa pensée. Elle reste toujours aussi neuve et ouverte, ne seraitce que par le stimulant que provoque la lecture de cette œuvre inépuisable. Elle ne connaît pas l'arrêt d'une interprétation globalisante. En échappant au système, d'où qu'il vienne, elle affirme son autonomie, sa capacité à la constante réinvention sémantique. De ce point de vue, l'œuvre de Nietzsche obéit à la dynamique d'une Aufhebung herméneutique. Loin de clore le débat qu'elle engendre, l'interprétation le déplace, l'approfondit et finit par lui donner une nouvelle dimension qui l'ouvre à de nouveaux horizons. Elle suit les méandres d'une existentialité tourmentée d'elle-même. Ce qui lui donne sa tonalité tragique, ce pessimisme des forts, selon l'expression de Nietzsche lui-même.

2 L'œuvre de Nietzsche ne se développe pas comme une doctrine ou un système, même si elle comporte des arêtes extrêmement précises comme la volonté de puissance, l'éternel retour ou l'amor fati. On ne la réduira pas davantage aux analyses suggestives qu'elle contient comme la différence fondamentale entre libre penseur et esprit libre ou celle entre polythéisme et monothéisme. Le décisif en ce qui concerne Nietzsche, se trouve dans cet " autrement penser ", " anders denken ». Penser ce n'est jamais répéter, redire dans une stricte continuité logique. La répétition est un mécanisme de la vitalité et non une modalité du penser. Si l'homme est bien un vivant, cet existant pensant interrompt la répétitivité vitale dans la distance réflexive. Penser, c'est imposer une rupture dans l'immédiat compulsif du vivant; il faut récompenser ce qu'advient par la vie. Dans la mesure où l'œuvre de Nietzsche se veut être ce " penser autrement » chacun se retrouve dans sa réflexion, non pour s'y complaire, mais pour être renvoyé à ses propres ruptures. N'est-ce pas cela qui fait l'attrait d'une pensée qui advient à chacun, mais n'identifie personne ? Un peu à l'image de ce que Nietzsche dit de son Ainsi parlait Zarathoustra : un livre pour tous et pour personne. Les pages qui suivent devraient, à leur façon, illustrer cette attirance de la pensée nietzschéenne sur des auteurs aux parcours bien différentiés. 
3 Dans le cadre d'un colloque organisé par le Centre Autonome d'Enseignement de Pédagogie religieuse et la section " Philosophie » de l'Université de Metz en septembre 2000, deux propositions nietzschéennes avaient été soumises à réflexion. Au dire de Nietzsche lui-même, l'une comme l'autre se veulent provocantes pour appeler le débat, la controverse inhérente à l'autrement du penser nietzschéen. S'il est vrai qu'on ne débat que de ce que l'on combat - sinon tout est répétition -, l'ambiguïté de la proposition est ce qui suscite l'intérêt. Non dans un besoin de simplifier mais d'approfondie, d'aller au fondement nocturne qui est l'autrement d'un penser qui se prétend clairvoyant.

4 La première affirmation se trouve dès le départ de la trajectoire nietzschéenne dont elle sert, en quelque sorte, de fil conducteur puisqu'elle est constamment reprise : « L'existence et l'univers ne sont éternellement justifiés qu'en tant que phénomène esthétique ». La deuxième, postérieure de quelques années, apparaît explicitement dans le Gai Savoir : " Dieu est mort ; mais tels sont les hommes qu'il y aura peut-être encore pendant des millénaires des cavernes dans lesquelles on montrera son ombre ».

On pourrait penser que ces deux propositions sont irréductibles l'une à l'autre et ne permettent aucune passerelle significative. En est-on bien sûr ? En réalité la mort de Dieu, en effaçant le monde de la transcendance et des valeurs dites supérieures, selon l'expression même de Nietzsche, confirment l'affirmation première et constante : tout se joue, du point de vue de l'existence humaine, dans la sphère esthétique c'est-à-dire dans l'ordre du sensible. L'aisthesis est bien, aux yeux de Nietzsche, l'unique patrie de l'homme, celle que chante Zarathoustra qui invite ses fidèles, les hommes, à y rester fidèles ! La mise en valeur de ce patrimoine exige la mort de Dieu.

6 Le champ existentiel nietzschéen perd ainsi de la hauteur, de cette hauteur qui est la demeure symbolique du divin transcendant. Mais en revanche, il gagne en profondeur pour inventorier les zones obscures de la vitalité où rayonne « le gracieux soleil de la Nuit » comme le chante Novalis dans ses Hymnes à la Nuit. Car la nuit révèle cet autre absolu, impersonnel, qui emporte l'individu dans les ivresses dépersonnalisantes du vital. Nietzsche est sensible à ce dynamisme pulsionnel, véritable " es " primal dont Freud s'efforcera de décrypter les mécanismes. Nietzsche est celui qui le nomme et en fait le véritable acteur du penser. Car note-t-il, « une pensée vient quand elle veut et non quand je veux » . Le " es » dessine la profondeur, l'épaisseur du champ humain dans lequel s'affrontent les dynamismes, les forces de la vitalité. La mort de Dieu comme l'effacement de l'être n'ont d'autre but que de perᄀmettre à la vitalité de fonctionner selon la logique de la puissance. La formule nietzschéenne pour définir cette vitalité sera donc volonté de puissance. Ce " vouloir de puissance " identifie la vitalité et signifie sa valeur. « Il n'est rien dans la vie qui ait de la valeur, sinon le degré de puissance - à supposer justement que la vie elle-même soit volonté de puissance " . De ce primat reconnu à la puissance même de la vie découle le refus nietzschéen de toute évaluation morale qui en perturberait le fonctionnement.

7 S'il est vrai que la philosophie de Nietzsche trouve dans ce "vouloir de puissance » la formule même de la vie, elle scelle le destin de l'homme en le soumettant aux dynamismes aveugles de la force. On est en droit de s'interroger sur la signification anthropologique comme sur la pertinence éthique d'une telle problématique. Car la puissance (Macht) a aujourd'hui une telle capacité de «faire » (machen) qu'elle est en mesure de « dé-faire » le dispositif élémentaire du monde et de ceux qui y naissent, y 
grandissent et y meurent ! Ce pouvoir-faire, quasi illimité, peut-il être sa propre normalité, au point de laisser le loup dévorer l'agneau et le plus fort dominer le faible?

Cette question ne peut pas ne pas être posée. À partir de ce que le xxe siècle a connu d'entreprises déshumanisantes, nous savons que la puissance, de par sa propre logique, est aliénante du point de vue anthropologique. Car, par définition, elle nie la liberté. La puissance impose une relation de force, de conquête qui ne respecte pas l'autre dans son intégrité. Elle a besoin, pour manifester sa force, d'un adversaire qu'elle se soumet. Il lui faut un vis-à-vis à dominer. Comment un tel processus sans liberté pourrait-il devenir le mode de relations inter-humaines ? Est-il seulement pensable de définir un espace humain sans liberté, à partir des seuls mécanismes de la domination ? Impossible.

Dans sa réflexion sur Auschwitz, Hans Jonas en vient à poser la question de la pertinence du Dieu biblique, qualifié de Tout-Puissant. Si la puissance, argument-il, réduit à néant la condition d'homme, si elle est une machine à humiliation, à défiguration humaines, si elle efface l'image même du divin en l'homme, comment l'homme pourrait-il croire à ce Dieu Tout-Puissant ? Si le qualificatif de puissance qui sert à désigner le Dieu biblique devient synonyme de mal par excellence, n'y a-t-il pas quelque chose de perverti dans la notion même de divin?

S'il est légitime d'interroger la pertinence d'un divin dont il est dit qu'il est toutpuissant, à plus forte raison doit-on questionner le concept nietzschéen de "volonté de puissance » appliqués à la vie humaine si, par définition, il exclut la liberté et, de fait, incarne le mal ? En réduisant l'homme à ce "quelque chose " de la formule nietzschéenne pour le surhomme : «l'homme est quelque chose (etwas) qui doit être dépassé ", n'est-on pas entré dans une autre problématique nihiliste ? Tout se passe comme si la logique nihiliste de décadence que Nietzsche dénonce à propos de la transcendance métaphysique avait fini par atteindre l'homme lui-même. Si la vie se décline en termes de vouloir de puissance, ne rend-elle pas impossible un projet anthropologique de liberté ?

11 D'une façon ou d'une autre, les contributions que l'on trouve dans ce cahier, développe ce questionnement qui tourne autour du divin et donc de l'anthropologique. Le mérite de Nietzsche est de l'avoir initié sur des bases nouvelles qui rompaient avec la problématique métaphysique traditionnelle. Cette rupture a ouvert un nouveau champ au discours philosophique. Et le débat ne fait que commencer. 